\title{
The role of biomechanical forces in the natural history of
}

\section{coronary atherosclerosis}

Adam J. Brown MD, $P h D^{1}$, Zhongzhao Teng $P h D^{2}$, Paul C. Evans PhD ${ }^{3}$, Jonathan H. Gillard $M D^{2}$, Habib Samady $M D^{4}$ and Martin R. Bennett MD, $P h D^{1}$

${ }^{1}$ Division of Cardiovascular Medicine, University of Cambridge, UK

${ }^{2}$ Department of Radiology, University of Cambridge, UK

${ }^{3}$ Department of Cardiovascular Science, University of Sheffield, UK

${ }^{4}$ Department of Medicine, Emory University, Atlanta, USA

Short title: Biomechanical forces in coronary atherosclerosis

\section{Correspondence:}

Dr. Adam Brown

Division of Cardiovascular Medicine, University of Cambridge, Level 6, ACCI, Addenbrooke's Hospital, Cambridge, CB2 0QQ, UK

Tel: (44)1223 331504

Fax: (44)1223 331505

E-mail: ajdb2@cam.ac.uk

Total Word Count: 6,1325,968

Figures: 4

Tables: 2 


\begin{abstract}
Atherosclerosis remains a significant cause of morbidity and mortality worldwide, such that a thorough understanding of the underlying pathophysiological mechanisms is crucial if novel therapeutic strategies are to emerge. Although atherosclerosis is a systemic inflammatory disease, coronary atherosclerotic plaques are not uniformly distributed in the vascular tree. Experimental and clinical data highlight that biomechanical forces, including wall shear stress (WSS) and plaque structural stress (PSS), play a crucial role in the natural history of coronary atherosclerosis. Endothelial cell function is heavily influenced by changes in WSS and longitudinal animal and human studies have shown that coronary regions with low WSS undergo increased plaque growth. Local alterations in WSS may also promote transformation of stable to unstable plaque subtypes. Plaque rupture is determined by the balance between PSS and material strength, with plaque composition having a profound effect on PSS. Prospective clinical studies are now required to ascertain whether integrating mechanical parameters with medical imaging can improve our ability to identify patients at highest risk of rapid disease progression or sudden cardiac events.
\end{abstract}




\section{KEY POINTS}

- Atherosclerotic plaques are not uniformly distributed throughout the coronary tree, implying that local mechanical factors may determine plaque development and/or growth.

- Blood flow and particularly wall shear stress heavily influence endothelial function via diverse mechanisms.

- Biomechanical forces promote adverse changes in plaque composition, producing a 'high-risk' plaque phenotype that is more prone to rupture and sudden cardiac events.

- Plaque structural stress is determined by plaque composition, with plaque rupture occurring when plaque structural stress exceeds plaque strength.

- Prospective, observational studies suggest that integrating biomechanical parameters may improve our ability to identify patients at highest risk of rapid disease progression or plaque rupture. 


\section{INTRODUCTION}

Ischaemic heart disease remains a leading cause of mortality and morbidity throughout the world. The development of atherosclerotic plaques within coronary arteries may give rise to a number of adverse clinical events, including sudden cardiac death and myocardial infarction (MI). Although the incidence of MI continues to decrease in developed countries as a consequence of lifestyle improvements, risk factor modification and therapeutic advances ${ }^{1}$, the number of coronary events in developing and newly industrialised countries is rising ${ }^{2}$. Thus, a thorough understanding of the pathological mechanisms underlying atherosclerotic plaque initiation, development and failure is critically important if new diagnostic and therapeutic strategies are to successfully emerge.

Atherosclerosis predominantly affects the intimal layer of the arterial vessel wall and is characterised by deposition of extracellular lipids and inward migration of pro-inflammatory bone marrow-derived cells and proliferation and migration of local smooth muscle cells ${ }^{3}$. The initial pathological cellular changes associated with atherosclerosis can develop in early childhood, but overt macroscopic atherosclerotic plaques are rarely observed before the second or third decade of life ${ }^{4}$. Although atherosclerosis is a systemic disease that may affect several arterial territories at once, plaques are focally distributed within the coronary tree and individual lesions are often observed at different stages of development. Indeed, plaque evolution does not occur in a linear manner, with some plaques transforming into advanced lesions with large lipid necrotic cores and thin overlying fibrous caps (a 'thin-cap atheroma' (TCFA)), while others regress and become less likely to result in clinical events ${ }^{5,6}$. While the physical substrate for plaque rupture has been defined from post-mortem studies, prediction of rupture prior to the event is a significant diagnostic challenge, with medical imaging based on plaque anatomy providing only limited ability to predict future cardiovascular events ${ }^{7-9}$. 
The suggestion that local environmental factors influence plaque development and behaviour means that novel modalities that can measure these factors may complement plaque imaging and improve our ability to predict future events.

The non-uniformity in atherosclerosis distribution within an individual and difficulty in predicting plaque behaviour from imaging alone may be explained, in part, through study of the biomechanical forces acting within the artery. For example, mechanical forces play an important role in cellular physiology ${ }^{10}$, resulting in pro-atherogenic changes within the endothelium that ultimately influence the macroscopic and microscopic architecture of the plaque, which in turn regulates plaque development. These changes may also weaken the plaque, resulting in plaque failure ${ }^{11}$. We describe and review those biomechanical forces that act within coronary arteries and explore their mechanisms and role in the natural history of coronary atherosclerosis, both in animal models and human studies. Calculation and integration of biomechanical parameters may improve our ability to identify arterial regions at risk of atherosclerosis, allowing better identification of patients at the highest risk of clinical events.

\section{BIOMECHANICAL FORCES}

Coronary arteries are continually subjected to biomechanical forces during each cardiac cycle, due to the pulsatile pressure of blood passing from the aorta through the coronary tree. These complex mechanical forces act in circumferential, radial and axial directions, deforming individual cells, atherosclerotic tissue and the artery wall to create stresses. In biomechanics, a stress is defined as the force acting on a surface, divided by the area of that surface. Although exact measurement of coronary stresses in vivo is challenging, they can be estimated through advanced computational techniques. In the context of cardiovascular 
research, the two important stresses that have been hypothesised to affect the natural history of atherosclerosis are wall (or endothelial) shear stress (WSS) and plaque structural stress (PSS) (Table 1).

\section{Wall shear stress}

WSS is the parallel frictional force exerted by blood flow on the endoluminal surface of the arterial wall. Magnitude of WSS is expressed using a variety of interchangeable units (e.g. 1 $\mathrm{Pa}=1 \mathrm{~N} / \mathrm{m}^{2}=10$ dynes $/ \mathrm{cm}^{2}$ ), with values being influenced by changes in luminal geometry, blood flow velocity and plasma viscosity. WSS is dependent on anatomical location, with the highest values often observed in the distal vessel or in arterial side-branches ${ }^{12}$. WSS is also very sensitive to pre-existing arterial geometry, being increased at the outer curvature and reduced at the inner curvature of the vessel wall ${ }^{13,14}$. If blood flow rate is constant, a stenotic atherosclerotic plaque will significantly increase WSS at the site of maximal stenosis, as blood becomes accelerated through a smaller, cross-sectional luminal area. In contrast, disturbed blood flow, defined as a pattern of flow that is non-uniform and irregular resulting in eddies and changes to flow direction ${ }^{15}$, can be induced by arterial bifurcations, vessel curvature and typically observed downstream of a stenotic plaque ${ }^{16}$ (Figure 1).

WSS can be estimated in vivo through computational fluid dynamics (CFD) simulations, a numerical method that mathematically approximates the flow fields of liquids within a structure. Several factors first need to be defined to perform CFD analysis, including 1) an accurate 3D geometry of the coronary arteries created from imaging, 2) coronary physiological parameters, such as coronary blood flow velocity and pressure, forming the loading conditions for the simulation and, 3) numerical solutions for the physical laws governing the motion and behaviour of blood (Figure 2). Although these basic elements are 
required for WSS calculations, there remains ongoing debate and research regarding the optimal appropriate methodology ${ }^{17-19}$. For example, recent data supports the use of 'true' anatomical coronary models, with blood flow also being modelled through side branches ${ }^{20}$. Nevertheless, this lack of a standardised approach for computational modelling has introduced heterogeneity in the metrics used to quantify and describe WSS in atherosclerosis, leading to some challenges in data interpretation between studies ${ }^{21}$.

\section{Plaque structural stress}

PSS is the stress located within the body of an atherosclerotic plaque or the arterial wall as a consequence of vessel expansion and stretch induced by arterial pressure. However, PSS is also determined partially by plaque composition/morphology and the material properties of tissues. Synonymous terms include 'tensile stress', 'structural stress' or even 'biomechanical stress'. PSS is typically around $10^{3}-10^{5}$ times greater than WSS in humans and often expressed in kilopascals $(\mathrm{kPa})^{22}$. PSS correlates positively with vessel luminal area in a vessel of uniform wall thickness and pressure, such that higher PSS values are expected in the proximal segments of coronary arteries. In contrast, lower PSS values are expected at stenoses and with increasing vessel wall thickness PSS $^{23}$. However, changes in composition and architecture of the plaque and artery wall can alter PSS significantly ${ }^{24}$. For example, PSS is markedly affected by the juxtaposition of tissues of different mechanical properties. Increased PSS levels may be a principal mechanism through which plaques rupture, resulting in thrombosis and sudden ischemic clinical events ${ }^{25}$. Localised high PSS concentrations may also affect cellular function, modifying the structural integrity of the plaque ${ }^{26}$ (Figure 1).

Estimation of PSS can be performed using structural mechanical techniques, including finite element analysis (FEA); a numerical method that mathematically models the effect of a force 
being applied to a structure. FEA allows an approximation of the solution by first subdividing the structure being studied into many (often >10,000) smaller elements. The FEA solution first requires a model of the coronary artery, which is typically generated from a highresolution imaging modality (e.g. intravascular ultrasound). The model must accurately depict both atherosclerotic lesion composition and architecture, as FEA simulations are sensitive to subtle changes in plaque or luminal geometry. Coronary arterial pressure and the material properties of each atherosclerotic plaque component and arterial wall are required to simulate lesion deformation. However, data on the material properties of atherosclerotic tissue are minimal and there is likely a variation between different patients and even plaque subtypes $^{27}$, which has potential to affect overall PSS calculations ${ }^{28}$. At present, the majority of studies use the material properties derived from ex vivo tensile testing of plaque samples ${ }^{29-}$ ${ }^{31}$, although research using patient-specific material properties is ongoing. Once complete, the final solution can allow estimation of either maximum principal stress and/or von Mises stresses, two differing engineering measures that can be used to predict whether any specific material is prone to structural failure ${ }^{32}($ Figure 3).

Alternatively, PSS can be calculated through fluid-structure interaction (FSI) simulations, an approach that seeks to integrate both the fluid and structural mechanical forces into a defined solution $^{33}$. Although FSI allows quantification of both WSS and PSS within an individual artery, the engineering processes involved are increasingly complex. The increased analytical demand of FSI modelling limits its current application either to idealised models or small clinical studies. Furthermore, it is unclear whether the small incremental gain in accuracy achieved by FSI simulations is clinically meaningful, when compared with computationally analyses that are less time consuming ${ }^{34}$. 


\section{BIOMECHANICAL FORCES INVOLVED IN PLAQUE INITIATION}

Atherosclerotic plaques are not uniformly distributed throughout the coronary tree ${ }^{35,} 36$. Biomechanical forces influence the initiation of atherosclerosis, with plaques developing predominantly near to side branches and/or bends in arteries. Blood flow at these sites is disturbed due to the uneven luminal geometry, leading to alterations in both the magnitude and direction of WSS. Arterial regions exposed to low time-averaged WSS or to patterns of WSS that change direction (oscillatory or tangential) appear prone to atherosclerosis, whereas regions exposed to WSS with higher magnitude and uniform direction are protected ${ }^{16,21,37}$. However, there may be a ceiling at which WSS loses its atheroprotective effects, with studies showing that supraphysiological WSS may also modify the endothelial response to promote plaque initiation $^{38}$.

A causal relationship between WSS and initiation of atherosclerosis has been established by studies where arterial flow is altered in experimental animals either by an extravascular $\operatorname{cuff}^{39,40}$, partial ligation, or tandem ligations of the carotid artery ${ }^{41}$. These studies revealed that although low WSS per se is not sufficient to induce atherosclerosis, it induces focal endothelial cell (EC) dysfunction and inflammation, which primes arterial regions for subsequent atherosclerosis initiation in response to hypercholesterolaemia. In addition, as magnitude of WSS varies with lumen remodelling, ECs overlying plaques are exposed to much higher levels compared to healthy endothelium ${ }^{42}$. Intriguingly, plaque rupture localises preferentially to the upstream portion of the plaque that is exposed to the highest levels of $\mathrm{WSS}^{43}$. The causal role of WSS in atherosclerosis is reinforced by studies showing that higher time-averaged WSS is associated with atheroprotection in healthy arteries, whereas supraphysiological levels of WSS correlate with EC injury and may be associated with 
plaque instability. One possible explanation for these observations is that physiological and supraphysiological levels of WSS may have differing effects on EC function (see below).

\section{Endothelial cell behaviour is affected by changes in blood flow}

EC at atheroprone sites exposed to disturbed flow exhibit highly heterogenous and often contradictory behaviour $^{16,44}$ (Figure 1). For example, disturbed flow induces EC apoptosis via activation of $\mathrm{JNK}^{45}, \mathrm{p} 53^{46}, \mathrm{PKC}^{47}$ and via the unfolded protein response ${ }^{48}$, and increases EC proliferation, which is also increased at atheroprone compared to atheroprotected sites ${ }^{45}$, 49 . In contrast, disturbed flow has also been reported to reduce EC proliferation via downregulation of the microRNA species miR-126-5 $\mathrm{p}^{50}$ and through the induction of irreversible cell cycle arrest (senescence) via p53-p21 signalling ${ }^{51}$. These studies suggest that atheroprone regions contain proliferating ECs as well as subpopulations in which the proliferative reserve has been exhausted, and that additional sub-populations undergo apoptosis. The processes that control differential cell fate decisions in ECs exposed to disturbed flow and the dynamic interactions between these different EC phenotypes should be studied further.

\section{Mechanotransduction modulates plaque inflammatory signalling}

The initiation of atherosclerosis involves the recruitment of circulating leukocytes to the vessel wall. This highly co-ordinated process, termed the leukocyte adhesion cascade, involves capture of leukocytes by activated ECs and subsequent rolling and transmigration into underlying vascular tissues. Monocyte migration is promoted by several proinflammatory mediators including cytokines (e.g. TNF $\alpha$, IL-1) and oxidized lipoproteins. WSS has profound effects on inflammatory activation of ECs (Figure 1), such that higher uniform WSS reduces subsequent induction of VCAM-1 and E-selectin expression in ECs in culture $^{52,53}$ and adhesion of monocytes ${ }^{54,55}$. In contrast, low oscillatory WSS promotes 
monocyte adhesion by enhancing expression of inflammatory adhesion molecules, including JAM-A ${ }^{54,56,57}$. Consistent with these in vitro observations, en face staining of arteries demonstrates that atheroprone regions exposed to low WSS are more inflamed than sites exposed to high $\mathrm{WSS}^{58,59}$.

Flow regulates EC inflammatory activation by altering activity of a number of inflammatory pathways including NF- $\mathrm{BB}$ and MAP kinase signalling (Figure 4). In unstimulated cells, NF$\kappa \mathrm{B}$ is held in the cytoplasm by its inhibitor I $\mathrm{KB}$. Inflammatory mediators induce phosphorylation and degradation of $\mathrm{I} \kappa \mathrm{B}$, leading to nuclear translocation and activation of $\mathrm{NF}-\kappa \mathrm{B}$. The induction of inflammatory genes by NF- $\kappa \mathrm{B}$ is enhanced in EC exposed to low oscillatory WSS compared to higher uniform $\mathrm{WSS}^{58}$. The mechanism relies on increased expression of RelA NF- $\kappa$ B subunits, whose transcription is increased by low WSS via a JNKATF2 pathway ${ }^{59}$. Although NF- $\kappa \mathrm{B}$ expression is increased by low WSS it remains cytoplasmic and inactive in the majority of cells. Thus, increased NF- $\mathrm{B}$ expression primes EC for enhanced activation in response to a second inflammatory stimulus, such as hypercholesterolaemia ${ }^{58,59}$. Disturbed flow also promotes inflammation by suppressing the expression of MiR10a, a microRNA that negatively regulates canonical NF- $\kappa$ B signalling ${ }^{60}$. In contrast, high uniform WSS reduces NF- $\mathrm{B}$ activity, which is mediated through several mechanisms including induction of KLF2, which inhibits NF- $\kappa \mathrm{B}$ transcription by sequestering the co-activator $\mathrm{CBP} / \mathrm{p} 300^{61}$, and activation of endothelial nitric oxide synthase (eNOS), which generates NO to suppress I $\kappa \mathrm{B}$ kinase, a positive regulator of NF- $\kappa \mathrm{B}^{62}$. High uniform flow also alters the function of NF- $\kappa B$ by enhancing NF- $\kappa B-d e p e n d e n t$ cytoprotective and anti-inflammatory responses and simultaneously inhibiting NF- $\mathrm{B}$ dependent inflammation ${ }^{63}$. 
WSS also regulates the activity of the pro-inflammatory MAP kinases JNK and p38 and downstream AP-1 family transcription factors. The mechanisms that link WSS with MAP kinases involve KLF2, which is induced by high WSS and subsequently inhibits phosphorylation and nuclear localization of AP-1 family members c-Jun and ATF2 ${ }^{64-66}$. High WSS also activates the transcription factor $\mathrm{Nrf} 2^{67-69}$ which function together with MAP kinase phosphatase- 1 to reduce inflammation by dephosphorylating p38 and $\mathrm{JNK}^{69,70}$. The inhibitory effect of high WSS on inflammatory MAP kinases is also mediated via downregulation of thioredoxin interacting protein (TXNIP) leading to inhibition of apoptosis signal-regulating kinase 1 , a kinase that acts upstream of p38 and $\mathrm{JNK}^{71}$.

Although higher or physiological WSS reduces inflammation, supraphysiological WSS or very high WSS can have the opposite effects. This was demonstrated using a murine model of arteriovenous fistula that generates enhanced WSS in the carotid artery ${ }^{72}$. Increased WSS induced activation of $\mathrm{NF}-\kappa \mathrm{B}$ and the expression of matrix metalloproteinases involved in inflammation and vascular remodelling. In vitro studies demonstrated that supraphysiological WSS (7.5 Pa) enhanced phosphorylation of p38, c-Jun and ATF2 compared to physiological WSS $(1.5 \mathrm{~Pa})^{38}$, suggesting that supraphysiological WSS may contribute to plaque instability by triggering inflammation and/or EC erosion.

\section{BIOMECHANICAL FORCES INVOLVED IN PLAQUE GROWTH}

\section{Plaque growth and arterial remodelling}

There is increasing literature in both animals and humans demonstrating that WSS can affect both plaque growth and transformation into high-risk plaques. For example, atherosclerotic plaques responsible for $\mathrm{MI}$ are more frequent within proximal vessels or around bifurcations $^{73,74}$. Low WSS regions are present within proximal vessels and on the 
contralateral wall to arterial side-branch divisions ${ }^{12}$. Animal studies using a partial arterial ligation model showed that co-localisation of low and oscillatory WSS acted synergistically to promote atherogenesis ${ }^{75}$, whereas low WSS was associated with plaque burden and positive remodelling ${ }^{76}$. Similarly, the spatial and temporal relationship between low WSS and plaque progression was illustrated through longitudinal animal studies. Here, arterial segments with low WSS at baseline exhibited greater subsequent plaque progression compared with moderate/high WSS regions ${ }^{77}$. Furthermore, low WSS was frequently associated with excessive expansive remodelling, a form of compensatory remodelling where both lumen and vessel dimensions increase. These changes to the arterial structure act to decrease WSS within these segments, further driving rapid plaque progression ${ }^{78}$. This was in contrast to regions with negative (or constrictive) remodelling, where WSS was increased and plaque growth appeared almost attenuated.

The relationship between baseline WSS and plaque progression has also been found in humans (Table 2). The potential for in vivo WSS calculations was first demonstrated using a combination of coronary angiography and intravascular ultrasound (IVUS) to reconstruct luminal geometry ${ }^{79}$, before being confirmed in a longitudinal study of twelve patients who had one coronary artery with a $<50 \%$ obstruction $^{80}$. Investigators observed significant increases in plaque thickness associated with regions of low WSS, which was again coupled with expansive remodelling, mirroring the results observed in animal models. However, in humans there is greater uncertainty over the relationship between WSS and vascular remodelling, as both constrictive remodelling and eccentric plaque development have also been observed ${ }^{81,82}$. Other studies have reported contradictory findings and observed that high WSS regions were more likely to result in excessive expansive remodelling ${ }^{83}$. Notably, plaque growth was predicted better when WSS calculations were blended with established 
imaging features known to predict disease progression ${ }^{84}$. The translational potential of baseline WSS calculations was tested in the PREDICTION study, which used coronary angiography and intravascular ultrasound (IVUS) to reconstruct 1,341 arteries from 506 patients undergoing percutaneous coronary intervention following an acute coronary syndrome ${ }^{85}$. Increases in plaque area were independently predicted by plaque burden, as quantified by IVUS, with decreasing luminal area (analogous to constrictive remodelling) predicted by low WSS. Additionally, both increased plaque burden $(\geq 58 \%)$ and low WSS $(<0.98 \mathrm{~Pa})$ were associated with further luminal obstruction necessitating repeat coronary intervention. Further longitudinal studies are now warranted to assess whether integration of WSS calculations with anatomical imaging can better predict future clinical events. This may be increasingly possible through the use of improvements in non-invasive coronary imaging, allowing the construction of 3D arterial geometry with relative ease $\mathrm{e}^{86-88}$.

\section{Transformation into high-risk plaques}

Coronary plaques responsible for around two-thirds of acute coronary events, including sudden cardiac death and MI, are lipid-rich and frequently have a thin overlying fibrous cap ${ }^{89}$, 90. A hypothesised precursor lesion has been proposed, the thin-cap fibroatheroma (TCFA), which shares several of these characteristics and may also display additional structural and compositional features that act to induce instability (Box 1) ${ }^{91}$. However, post-mortem and clinical studies have shown that TCFA are not distributed evenly throughout the coronary $\operatorname{arteries}^{35,92}$, while longitudinal clinical studies have shown that stable plaques may evolve and transform into TCFA with the passage of time ${ }^{5,93}$. Local alterations in WSS have been suggested as one mechanism that may be involved in this dynamic process. 
Animal and ex vivo studies provide the majority of data linking alterations in WSS to changes in plaque composition. Regions of low WSS correlate with increasing lipid accumulation within the arterial wall ${ }^{94,95}$, possibly through inducing conformational changes in ECs that act to increase permeability to low-density lipoproteins ${ }^{96,97}$ or though up regulation of genes encoding the LDL receptor ${ }^{98,99}$. This crucial pro-atherogenic effect of low WSS itself appears to be affected by total serum cholesterol levels, with low WSS and high cholesterol acting synergistically to promote increased plaque growth, lipid accumulation and leukocyte infiltration ${ }^{100}$. Smooth muscle cell migration and apoptosis may also be affected by changes to $\mathrm{WSS}^{101,102}$, resulting in regions of persistently low WSS having reduced collagen content ${ }^{94}$ and being associated with fibrous cap thinning ${ }^{103}$. WSS has also been linked with increased plaque inflammatory activity and increased expression of MMPs ${ }^{104}$, which act to reduce plaque strength through degradation of extracellular matrix proteins ${ }^{105}$. Finally, longitudinal studies in porcine models have shown that WSS changes dynamically over time, with TCFA developing more frequently in coronary regions exposed to low WSS throughout their evolution $^{103,106}$.

Assessing the relationship in humans between WSS and coronary plaque composition has proven challenging, as non-invasive imaging modalities currently struggle to characterise plaque morphology in sufficient detail for subtle changes in composition to be studied ${ }^{107-109}$. Thus, the majority of human data on WSS have been obtained through use of invasive imaging modalities, including virtual-histology (VH)-IVUS ${ }^{110}$ or optical coherence tomography $(\mathrm{OCT})^{111}$. In a study of twenty patients low WSS regions were found to develop greater plaque and necrotic core progression over 6 months when compared with intermediate WSS regions ${ }^{83}$. Indeed, a negative relationship between WSS and lipid accumulation has been found to exist, with a $17 \%$ increase in necrotic core for every 1 Pa decrease in $\mathrm{WSS}^{112}$. 
A spatial relationship between low WSS regions and necrotic core was observed in early plaques (plaque burden $<40 \%$ ) imaged by VH-IVUS ${ }^{113}$, while increases in necrotic core percentage occured at the ostium of the left anterior descending artery, an anatomical site typically affected by low WSS values ${ }^{114}$. WSS has also been calculated in patients presenting with an acute coronary syndrome imaged by OCT, with low WSS regions having a higher prevalence of macrophages, lipid-rich plaques and OCT-defined TCFA ${ }^{115}$. Furthermore, fibrous cap thickness was reduced in low WSS segments, with these coronary regions showing more superficial calcification.

\section{BIOMECHANICAL FORCES INVOLVED IN PLAQUE FAILURE}

The structural failure of the majority of atherosclerotic plaques is due to rupture of the fibrous cap $^{116}$, while repeated cycles of rupture and repair are thought to drive rapid plaque progression and growth ${ }^{117}$. Post-mortem studies have shown that ruptured plaques exhibit specific compositional features, including a large necrotic lipid core, macrophage infiltration and microcalcification, and an overlying fibrous cap with less collagen and fewer smooth muscle cells than stable lesions. Significant attempts have been made to identify plaques with unstable features in vivo, yet prospective studies have shown that few plaques labelled as 'high-risk' for rupture actually result in adverse clinical events ${ }^{7-9}$. Thus, rupture is not solely dependent on plaque morphology, and other local factors are likely involved. In recent years, there has been a resurgence of interest in the role of biomechanical forces in plaque rupture, as a consequence of improvements both in the imaging of coronary atherosclerosis and in computational power. 


\section{Experimental data}

Increased PSS has long been hypothesised as a fundamental mechanism that precipitates rupture of a vulnerable plaque ${ }^{25}$, as alterations to either plaque architecture or composition significantly affect PSS values. In idealised models of coronary plaques, PSS is increased with increased necrotic core thickness/size, during early stages of positive remodelling, when luminal curvature is increased (as would be expected at the plaque shoulder) and when fibrous cap thickness is reduced ${ }^{24,118,119}$. 3D idealised plaque models have confirmed these findings, where a reduction in fibrous cap thickness by $50 \%$ results in a $30 \%$ increase in $\mathrm{PSS}^{120}$. The thickness of the fibrous cap may be the principal determinant of PSS, as PSS values appear more sensitive to changes in fibrous cap thickness than necrotic core volume $^{121}$. For example, PSS can exceed $300 \mathrm{kPa}$ if fibrous cap thickness is $<60 \mu \mathrm{m}$, a value whereby the cap is prone to failure ${ }^{122}$.

Another key determinant of PSS is the location, extent and size of individual calcium deposits within the plaque. For example, superficial calcification around or within the fibrous cap increases PSS by almost $50 \%^{123}$. In addition, whereas larger plates of calcification (generally those $>1 \mathrm{~mm}$ in size) may be a plaque stabilising feature by FEA models ${ }^{124}$, small foci of calcium significantly increase $\mathrm{PSS}^{125}$. In a study using idealised 3D models, maximal PSS values were not located on the calcium itself, but instead occurred just upstream ${ }^{125}$, suggesting that calcium may focus high stress concentrations on tissues that may not have the mechanical strength to withstand these high stress values. Finally, microscopic, cellular-level microcalcifications $(\sim 5-10 \mu \mathrm{m}$ diameter $)$ have been shown to be common and accumulate within either apoptotic smooth muscle cells or macrophages located in the fibrous cap ${ }^{126}$. When these minute particles of calcium were clustered together along the tensile axis of the cap, they could increase local PSS by a factor of $>5^{127}$. This pattern was found to occur very 
infrequently however, which may explain why so few TCFA progress to rupture in clinical studies. Intriguingly, recent research has shown that microcalcification may become be identified through use of novel imaging techniques, including ${ }^{18} \mathrm{~F}$-sodium fluoride positron emission tomography ${ }^{128-130}$.

\section{Clinical data to support role of PSS in rupture}

Initial clinical studies examining the role of PSS in plaque rupture used histological plaque specimens as the geometrical basis for simulations. Here, PSS was found to be significantly increased in ruptured plaques responsible for sudden cardiac death when compared with stable lesions ${ }^{131}$. PSS levels in all ruptured plaques were found to exceed $300 \mathrm{kPa}$, and this PSS value was tentatively suggested as a predetermined 'threshold' for rupture. However, rupture is not always found to occur at peak PSS location, implying that regions of relatively high, rather than absolute peak stress, may be of equal importance. Indeed, high PSS regions were found to accurately predict plaque rupture location in $82 \%$ of lesions studied ${ }^{132}$. PSS may also directly alter plaque composition towards a more unstable subtype. For example, consistent with idealised experimental models, PSS increased as the necrotic core increased, with significant increases in PSS observed near the luminal surface of the plaque ${ }^{133}$. High PSS levels have been correlated with both increased MMP expression ${ }^{134}$ and macrophage accumulation $^{135}$, features known to weaken plaque mechanical strength. The regulation of vascular smooth muscle cells (VSMC) is also thought to be partly dependent on the degree of deformation (or stretch) within the artery wall ${ }^{136}$, with increased stretch promoting VSMC phenotypic switch from a contractile to a synthetic phenotype ${ }^{137}$. Inhibition of VSMC activity and stretch-induced VSMC apoptosis may also lead to reduced collagen content within the lesion, which again may weaken plaque structural integrity ${ }^{138}$. Lastly, high local stress concentrations were found around intra-plaque neovascularisation that displayed early signs 
of haemorrhage, suggesting that high PSS levels may trigger for neovessel rupture and intraplaque haemorrhage, with consequent potential for rapid plaque growth ${ }^{139}$.

Although histological analysis represents a useful tool to examine the composition and structure of atherosclerotic plaques, tissue processing can induce geometrical distortions that may affect the reliability of computational simulations ${ }^{140}$. Through improvement in medical imaging, it is increasingly possible to visualise coronary plaque morphology in vivo. Although most clinical studies of PSS in humans has been conducted in the carotid artery ${ }^{141-}$ ${ }^{143}$, a few studies have reported PSS calculations within the coronary tree using intravascular ultrasound (IVUS). Again, changes in plaque composition were found to modulate PSS, with increasing necrotic core elevating PSS, while increasing luminal stenosis attenuated values ${ }^{124}$. However, the relationship between calcification and PSS is more complex. While large plates of calcification appear to act as a 'stress-shield', tempering the effects of high PSS on the plaque, small calcific deposits may paradoxically increase PSS significantly, especially when they are in close proximity to one another ${ }^{127}$. IVUS was also used to create $3 \mathrm{D}$ models of the arterial wall in patients undergoing coronary intervention, with plaque rupture induced by balloon angioplasty ${ }^{144}$. The rupture site in this study co-localised with PSS location, demonstrating the feasibility of predicting rupture location. Finally, PSS calculated from 53 patients that underwent VH-IVUS before stenting demonstrated that stress values were highly heterogeneous throughout plaques, but increased values were observed in high-risk plaque regions in patients presenting with an acute coronary syndrome and within plaques classified as TCFA $^{145}$. 


\section{Alternatives mechanisms for rupture}

Observational studies have demonstrated that plaque rupture most frequently occurs at a proximal location (upstream) to the site of maximal stenosis ${ }^{146}$, a region where WSS is higher owing to increasing acceleration of blood through the luminal narrowing. This has led to the concept that increased WSS may promote plaque rupture ${ }^{147,148}$. In support of this, experimental studies have shown that increased WSS may induce erosion of the plaque surface $^{149}$ and promote platelet accumulation and thrombogenicity ${ }^{150,151}$, two factors that increase the risk of intraluminal thrombosis. Indeed, plaque erosion is considered the aetiological mechanism underlying around one-third of MI, particularly in younger patients, smokers and women ${ }^{152-154}$. Clinical studies too have shown that high WSS correlates with plaque rupture location, although imaging was typically performed after rupture had occurred $^{155}$. Finally, regions of high WSS and large plaque deformation have previously been found to co-localise ${ }^{156}$, meaning it may be difficult to disentangle whether it is increased WSS or PSS that drives rupture.

Although we have focussed particularly on WSS and PSS, material fatigue is another potential mechanism promoting plaque rupture ${ }^{157}$. Fatigue is the accumulation of microscopic levels of damage as a response to repeated loading. Although fatigue is widely accepted as a mechanism of structural failure in non-organic materials, biological tissues are thought to respond differently due to their inherent capability to heal or change their structure as a consequence of damage. Nevertheless, experimental models have suggested a role for fatigue in plaque rupture ${ }^{158}$, while a reduction in smooth muscle cells in the fibrous cap may inhibit the repair process, leading to accelerated rapid crack propagation and structural failure ${ }^{159,160}$. Further studies are now required to assess the response of atherosclerotic tissue to multiple repeated loading cycles that may precipitate material fatigue 


\section{CONCLUSIONS}

Biomechanical forces play a key role in the development of coronary atherosclerotic plaques through their effect on endothelial cell function. Mechanical stimuli also promote changes in plaque composition and plaque growth, with rupture governed by the dynamic interaction between plaque material strength and structural stress. Prospective clinical studies are now warranted to assess whether the integration of mechanical and medical imaging parameters improves our ability to identify those patients at highest risk of rapid disease progression or adverse clinical events.

\section{REVIEW CRITERIA}

Searches were performed on PubMed, MEDLINE and Central databases for full-text manuscripts published between 1990 and May 2015. The following search terms were used: "atherosclerosis", "coronary artery disease", "mechanotransduction", "shear stress", "endothelial stress", "wall stress", "tensile stress", "structural stress" and "plaque rupture". There were no limitations applied to language. 


\section{ACKNOWLEDGEMENTS}

This work was supported by the British Heart Foundation (FS/13/33/30168), Heart Research UK (RG2638/14/16), the Cambridge NIHR Biomedical Research Centre, and the BHF Cambridge Centre for Research Excellence. The authors would also like to thank Shuo Wang for preparation of images for publication.

\section{CONTIBUTIONS}

AJB, ZT and PC researched, wrote and critically edited the manuscript. JHG, HS and MRB reviewed, edited and added substantially to the scientific content presented.

\section{DISCLOSURES}

The authors have no competing interests to declare. 


\section{BIOGRAPHIES}

\section{Dr. Adam J. Brown}

Adam Brown is currently a British Heart Foundation Clinical Lecturer in Cardiology at the University of Cambridge and an Honorary Interventional Fellow at Papworth Hospital. He completed his undergraduate studies at the University of Cambridge and his early clinical training at both Addenbrooke's and Papworth Hospitals. His recently completed PhD focused on the use of intracoronary techniques, non-invasive imaging and mechanical modelling to identify and risk-stratify vulnerable coronary plaques.

\section{Dr. Zhongzhao Teng}

Zhongzhao Teng is Senior Research Associate at the Department of Radiology, University of Cambridge. He received his $\mathrm{BSc}$ and $\mathrm{PhD}$ from the Department of Mechanics and Engineering Science, Fudan University, Shanghai, China, in 1998 and 2003, respectively. In 2014, he completed his second doctorate at the Department of Radiology, University of Cambridge. Dr. Teng has a wide variety of research interests, including using combined invasive and non-invasive medical imaging modalities for carotid and coronary plaque imaging, integrating biomechanical engineering with medical imaging to improve clinical care and also exploring the material behaviour of biological tissues.

\section{Professor Paul C. Evans}

Paul Evans is Professor of Cardiovascular Science at the University of Sheffield. His research is focussed on the effects of shear stress on the physiology of vascular endothelial cells. These studies are relevant to vascular injury and atherosclerosis which develop predominantly at branches and bends in the arterial tree that are exposed to disturbed patterns 
of blood flow. The cross-disciplinary nature of his work involves interactions with engineers, physicists and mathematicians as well as molecular and cellular biologists and vascular physiologists.

\section{Professor Jonathan H. Gillard}

Jonathan Gillard is Professor of Neuroradiology at the University of Cambridge, an Honorary Consultant Neuroradiologist at Addenbrooke's Hospital and a Fellow of Christ's College, Cambridge. He undertook his undergraduate training in Guy's Hospital London, graduating in 1988, where he also gained a first class BSc in Radiological Sciences focusing on the use of transcranial ultrasound. His basic radiological training was undertaken in Cambridge; his specialist neuroradiological training was undertaken at Johns Hopkins Hospital and University in Baltimore. His 1997 MD was based on spectroscopic imaging in acute stroke. Professor Gillard now leads active research groups using MR methodologies in atheroma imaging, cardiovascular imaging and neuro-oncology He has published over 210 peer reviewed articles and has a longstanding interest in medical education, at an undergraduate and postgraduate level. He is also interested in management and received his MBA from the Cambridge Judge Business School in 2010.

\section{Professor Habib Samady}

Habib Samady is Professor of Medicine, Emory University School of Medicine, and Director, Cardiac Catheterization Laboratory, at Emory University Hospital. He is a physiologicallyoriented cardiologist and clinical investigator trained in general cardiology, nuclear cardiology and interventional cardiology at Yale University School of Medicine. In addition to patient care, Dr. Samady maintains an intensive research program: 1) hemodynamic mechanisms associated with progression of human coronary atherosclerosis; 2) evaluation of 
pharmaceutical agents for treatment of coronary microvascular disease, endothelial dysfunction, coronary vasospasm and non-obstructive atherosclerosis, and 3) biomechanics

of permanent and bioresorbable coronary vascular devices. He is Deputy Editor of JACC Cardiovascular Interventions and extensively published in the fields of intravascular imaging, coronary physiology, atherosclerosis assessment and stent biomechanics.

\section{Professor Martin R. Bennett}

Professor Bennett currently holds the British Heart Foundation Chair of Cardiovascular Sciences at the University of Cambridge, with Honorary Consultant Cardiologist positions at Addenbrooke's and Papworth Hospitals, and heads the Division of Cardiovascular Medicine in Cambridge. Professor Bennett directs the Cambridge component of the Oxbridge Centre for Cardiovascular Regenerative Medicine, and co-directs the Cambridge Cardiovascular Strategic Research Initiative and the Cambridge PhD programme in Cardiovascular Research. His major research interests are the vascular biology of atherosclerosis and invasive and noninvasive coronary artery imaging to identify vulnerable plaques. 


\section{TABLE LEGENDS}

\section{Table 1}

Descriptions of methodologies used and the biological impact of wall shear and plaque structural stresses

\section{Table 2}

Human prospective studies examining the relationship between baseline wall shear stress measures and changes to coronary plaque morphology 


\section{FIGURE LEGENDS}

\section{Figure 1 Biomechanical forces in atherosclerosis}

Alterations in both wall shear stress (WSS) and plaque structural stress (PSS) play important roles in coronary plaque development, progression and plaque rupture. Alterations in blood flow induced by geometrical changes to the arterial lumen result in low WSS regions, which acts to promote expression of inflammatory adhesion molecules and modifies endothelial cell turnover. In established lesions, subtle changes in plaque composition and/or architecture increase PSS, making the plaque more vulnerable to rupture.

EC, endothelial cell; JNK, c-Jun N-terminal kinases; p53, tumour protein p53; PSS, plaque structural stress; VCAM-1, vascular cell adhesion molecule-1; WSS, wall shear stress

\section{Figure 2. Wall shear stress calculations}

Invasive catheter angiography of a right coronary artery (RCA) at baseline showing an atherosclerotic coronary plaque (A). Repeat angiography for stable angina performed at 4 years $(\mathbf{B})$, showing a region of plaque progression with increase in luminal narrowing (arrow). Volume-rendered computerised tomography coronary angiogram of the RCA, again at baseline $(\mathbf{C})$, allowing reconstruction of the 3D geometry of the vessel (D). Computational fluid dynamic simulation $(\mathbf{E})$ based on the 3D geometry, showing a region of low wall shear stress that co-localised with the region of plaque progression (arrow). 


\section{Figure 3. Plaque structural stress calculations}

Virtual-histology intravascular ultrasound (VH-IVUS) imaging of a coronary atherosclerotic plaque detailing tissue composition and architecture (top panels). 3D model of the same coronary plaque (middle panel), providing the geometrical basis for finite element analysis. 3D band plot for plaque structural stress (PSS)(bottom panel), illustrating two regions of high PSS. Note the highest PSS value is located in close proximity to a region of calcification.

\section{Figure 4. Pro-inflammatory pathways regulated by mechanical sensing}

The influence of wall shear stress (WSS) on inflammatory signalling pathways is depicted. The effects of high (green) and low (red) wall shear stress (WSS) are indicated.

References are indicated.

ASK, apoptosis signal-regulating kinase; eNOS, endothelial nitric oxide synthase; IKK, I-kappa Bkinase; IL, interleukin; JNK, c-Jun N-terminal kinases; KLF2, Krüppel-like factor 2; MiR1Oa, microRNA10a; MKKs, mitogen-activated protein kinases; MKP-1, mitogen-activated protein kinase phosphatase-1; NF- $\kappa B$, nuclear factor kappa-light-chain-enhancer of activated B cells; Nrf2, nuclear factor erythroid-related factor-2; TAK, transforming growth factor beta-activated kinase; TNFR, tumour necrosis factor receptor; VCAM-1, vascular cell adhesion molecule-1 


\section{BOX 1}

Anatomical features of plaques at high risk of instability and rupture:

- Large, necrotic lipid core

- Thin overlying fibrous cap $\left(<65 \mu \mathrm{m}\right.$ on histology $\left.{ }^{91}\right)$

- Positive vascular remodelling

- Macrophage and inflammatory cellular infiltration of the fibrous cap

- Paucity of smooth muscle cells in the fibrous cap

- Plaque neovascularization

- Spotty calcification and low attenuation plaque (on CT scanning) ${ }^{161,162}$ 


\section{TABLE 1}

\begin{tabular}{|c|c|c|c|c|}
\hline Biomechanical stress & Scale & Computational method & Input parameters* & $\begin{array}{l}\text { Principal mechanism of } \\
\text { action in atherosclerosis }\end{array}$ \\
\hline \multirow[t]{2}{*}{$\begin{array}{l}\text { Blood flow-induced } \\
\text { wall shear stress (WSS) }\end{array}$} & \multirow[t]{2}{*}{ 1-10 Pa } & $\begin{array}{l}\text { Computational fluid mechanics } \\
\text { (CFD) }\end{array}$ & $\begin{array}{l}\text { Lumen boundary from imaging, } \\
\text { e.g., CT, IVUS, OCT }\end{array}$ & $\begin{array}{l}\text { Low WSS: atherogenesis } \\
\text { High WSS: atheroprotective }\end{array}$ \\
\hline & & $\begin{array}{l}\text { Fluid-structure interaction } \\
\text { (FSI) analysis }\end{array}$ & $\begin{array}{l}\text { Flow rate/velocity at the inlet and } \\
\text { pressures at outlets or pressures } \\
\text { at the inlet and outlets }\end{array}$ & $\begin{array}{l}\text { Supraphysiological WSS: } \\
\text { possibly involved in plaque } \\
\text { rupture or thrombus generation }\end{array}$ \\
\hline \multirow[t]{3}{*}{$\begin{array}{l}\text { Arterial pressure- } \\
\text { induced plaque } \\
\text { structural stress (PSS) }\end{array}$} & \multirow[t]{3}{*}{$\begin{array}{l}1-10 \times 10^{5} \\
\mathrm{~Pa}\end{array}$} & $\begin{array}{l}\text { Finite element analysis/method } \\
(\text { FEA/FEM }) \dagger\end{array}$ & $\begin{array}{l}\text { Detailed plaque structure from } \\
\text { high-resolution imaging, e.g. } \\
\text { IVUS }\end{array}$ & $\begin{array}{l}\text { High PSS: fibrous cap damage, } \\
\text { e.g., rupture }\end{array}$ \\
\hline & & \multirow[t]{2}{*}{ FSI analysis } & $\begin{array}{l}\text { Material properties of each } \\
\text { component }\end{array}$ & \multirow[t]{2}{*}{$\begin{array}{l}\text { Periodic change of PSS: fibrous } \\
\text { cap fatigue }\end{array}$} \\
\hline & & & $\begin{array}{l}\text { Flow rate/velocity at the inlet and } \\
\text { pressures at outlets or pressures } \\
\text { at the inlet and outlets }\end{array}$ & \\
\hline
\end{tabular}

\footnotetext{
* Inputs for WSS are for CFD and those for PSS are for the FSI analysis; + Except for structure and material properties, internal pressure is needed for FEA/FEM.
} 
TABLE 2

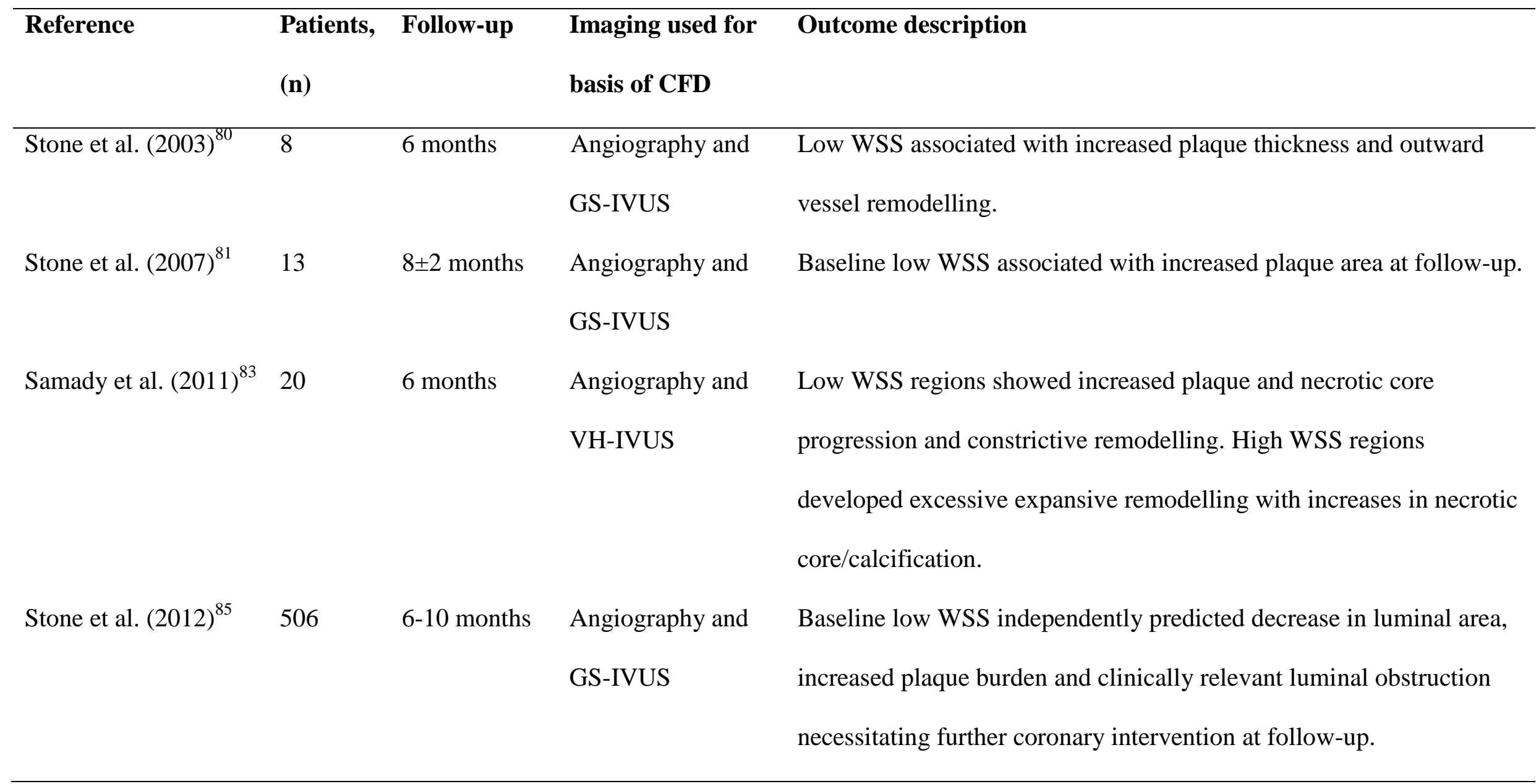

CFD, computational fluid dynamics; GS-IVUS, grey-scale intravascular ultrasound; OCT, optical coherence tomography; TCFA, thin-cap

fibroatheroma; WSS, wall shear stress; VH-IVUS, virtual-histology intravascular ultrasound 


\section{REFERENCES}

1. Yeh, R.W. et al. Population Trends in the Incidence and Outcomes of Acute Myocardial Infarction. New England Journal of Medicine 362, 2155-2165 (2010).

2. Celermajer, D.S., Chow, C.K., Marijon, E., Anstey, N.M. \& Woo, K.S. Cardiovascular Disease in the Developing WorldPrevalences, Patterns, and the Potential of Early Disease Detection. Journal of the American College of Cardiology 60, 1207-1216 (2012).

3. Libby, P., Ridker, P.M. \& Hansson, G.K. Progress and challenges in translating the biology of atherosclerosis. Nature 473, 317-325 (2011).

4. Stary, H.C. et al. A definition of initial, fatty streak, and intermediate lesions of atherosclerosis. A report from the Committee on Vascular Lesions of the Council on Arteriosclerosis, American Heart Association. Circulation 89, 2462-78 (1994).

5. Kubo, T. et al. The dynamic nature of coronary artery lesion morphology assessed by serial virtual histology intravascular ultrasound tissue characterization. Journal of the American College of Cardiology 55, 1590-7 (2010).

6. Raber, L. et al. Effect of high-intensity statin therapy on atherosclerosis in noninfarct-related coronary arteries (IBIS-4): a serial intravascular ultrasonography study. European Heart Journal 36, 490-500 (2015).

7. Calvert, P.A. et al. Association between IVUS findings and adverse outcomes in patients with coronary artery disease: the VIVA (VH-IVUS in Vulnerable Atherosclerosis) Study. JACC Cardiovascular Imaging 4, 894-901 (2011).

8. Stone, G.W. et al. A prospective natural-history study of coronary atherosclerosis. New England Journal of Medicine 364, 226-35 (2011). 
9. Cheng, J.M. et al. In vivo detection of high-risk coronary plaques by radiofrequency intravascular ultrasound and cardiovascular outcome: results of the ATHEROREMOIVUS study. European Heart Journal 35, 639-647 (2014).

10. Hahn, C. \& Schwartz, M.A. Mechanotransduction in vascular physiology and atherogenesis. Nature Reviews Molecular Cell Biology 10, 53-62 (2009).

11. Falk, E., Shah, P.K. \& Fuster, V. Coronary Plaque Disruption. Circulation 92, 657$671(1995)$.

12. Soulis, J.V., Farmakis, T.M., Giannoglou, G.D. \& Louridas, G.E. Wall shear stress in normal left coronary artery tree. Journal of Biomechanics 39, 742-749 (2006).

13. Zarins, C.K. et al. Carotid bifurcation atherosclerosis. Quantitative correlation of plaque localization with flow velocity profiles and wall shear stress. Circulation research 53, 502-14 (1983).

14. Ku, D.N., Giddens, D.P., Zarins, C.K. \& Glagov, S. Pulsatile flow and atherosclerosis in the human carotid bifurcation. Positive correlation between plaque location and low oscillating shear stress. Arteriosclerosis 5, 293-302 (1985).

15. Chiu, J.-J. \& Chien, S. Effects of Disturbed Flow on Vascular Endothelium: Pathophysiological Basis and Clinical Perspectives. Physiological Reviews 91, 327387 (2011).

16. Kwak, B.R. et al. Biomechanical factors in atherosclerosis: mechanisms and clinical implications. European Heart Journal 35, 3013-20, 3020a-3020d (2014).

17. Mejia, J., Mongrain, R. \& Bertrand, O.F. Accurate prediction of wall shear stress in a stented artery: newtonian versus non-newtonian models. J Biomech Eng 133, 4004408 (2011).

18. Timmins, L.H. et al. Focal association between wall shear stress and clinical coronary artery disease progression. Ann Biomed Eng 43, 94-106 (2015). 
19. Morbiducci, U. et al. On the importance of blood rheology for bulk flow in hemodynamic models of the carotid bifurcation. Journal of Biomechanics 44, 2427$2438(2011)$.

20. Li, Y. et al. Impact of Side Branch Modeling on Computation of Endothelial Shear Stress in Coronary Artery Disease: Coronary Tree Reconstruction by Fusion of 3D Angiography and OCT. Journal of the American College of Cardiology 66, 125-35 (2015).

21. Peiffer, V., Sherwin, S.J. \& Weinberg, P.D. Does low and oscillatory wall shear stress correlate spatially with early atherosclerosis? A systematic review. Cardiovascular research 99, 242-250 (2013).

22. Sadat, U., Teng, Z. \& Gillard, J.H. Biomechanical structural stresses of atherosclerotic plaques. Expert Review of Cardiovascular Therapy 8, 1469-81 (2010).

23. Fan, R. et al. Human coronary plaque wall thickness correlated positively with flow shear stress and negatively with plaque wall stress: an IVUS-based fluid-structure interaction multi-patient study. Biomed Eng Online 13, 13-32 (2014).

24. Ohayon, J. et al. Necrotic core thickness and positive arterial remodeling index: emergent biomechanical factors for evaluating the risk of plaque rupture. American Journal of Physiology - Heart and Circulatory Physiology 295, H717-H727 (2008).

25. Richardson, P.D., Davies, M.J. \& Born, G.V. Influence of plaque configuration and stress distribution on fissuring of coronary atherosclerotic plaques. Lancet 2, 941-4 (1989).

26. Wanjare, M., Agarwal, N. \& Gerecht, S. Biomechanical strain induces elastin and collagen production in human pluripotent stem cell derived vascular smooth muscle cells (2015). 
27. Akyildiz, A.C., Speelman, L. \& Gijsen, F.J.H. Mechanical properties of human atherosclerotic intima tissue. Journal of Biomechanics 47, 773-783 (2014).

28. Yuan, J. et al. Influence of material property variability on the mechanical behaviour of carotid atherosclerotic plaques: A 3D fluid-structure interaction analysis. International Journal for Numerical Methods in Biomedical Engineering 31 (2015).

29. Holzapfel, G.A., Sommer, G., Gasser, C.T. \& Regitnig, P. Determination of layerspecific mechanical properties of human coronary arteries with nonatherosclerotic intimal thickening and related constitutive modeling. Am J Physiol Heart Circ Physiol 289, 8 (2005).

30. Teng, Z. et al. Material properties of components in human carotid atherosclerotic plaques: A uniaxial extension study. Acta Biomaterialia 6, 00379-1 (2014).

31. Walsh, M.T. et al. Uniaxial tensile testing approaches for characterisation of atherosclerotic plaques. Journal of Biomechanics 47, 793-804 (2014).

32. Holzapfel, G.A., Mulvihill, J.J., Cunnane, E.M. \& Walsh, M.T. Computational approaches for analyzing the mechanics of atherosclerotic plaques: A review. Journal of Biomechanics 47, 859-869 (2014).

33. Yang, C. et al. In Vivo IVUS-Based 3-D Fluid-Structure Interaction Models With Cyclic Bending and Anisotropic Vessel Properties for Human Atherosclerotic Coronary Plaque Mechanical Analysis. IEEE transactions on bio-medical engineering 56, 2420-2428 (2009).

34. Huang, Y. et al. The influence of computational strategy on prediction of mechanical stress in carotid atherosclerotic plaques: Comparison of 2D structure-only, 3D structure-only, one-way and fully coupled fluid-structure interaction analyses. Journal of Biomechanics 47, 1465-1471 (2014). 
35. Cheruvu, P.K. et al. Frequency and distribution of thin-cap fibroatheroma and ruptured plaques in human coronary arteries: a pathologic study. Journal of the American College of Cardiology 50, 940-9 (2007).

36. Wykrzykowska, J.J. et al. Longitudinal distribution of plaque burden and necrotic core-rich plaques in nonculprit lesions of patients presenting with acute coronary syndromes. JACC Cardiovasc Imaging 5, 006 (2012).

37. Caro, C.G., Fitz-Gerald, J.M. \& Schroter, R.C. Arterial Wall Shear and Distribution of Early Atheroma in Man. Nature 223, 1159-1161 (1969).

38. White, S.J. et al. Characterization of the Differential Response of Endothelial Cells Exposed to Normal and Elevated Laminar Shear Stress. Journal of Cellular Physiology 226, 2841-2848 (2011).

39. Cheng, C. et al. Shear stress-induced changes in atherosclerotic plaque composition are modulated by chemokines. Journal of Clinical Investigation 117, 616-626 (2007).

40. Cheng, C. et al. Atherosclerotic lesion size and vulnerability are determined by patterns of fluid shear stress. Circulation 113, 2744-2753 (2006).

41. Nam, D. et al. Partial carotid ligation is a model of acutely induced disturbed flow, leading to rapid endothelial dysfunction and atherosclerosis. American Journal of Physiology-Heart and Circulatory Physiology 297, H1535-H1543 (2009).

42. Pedrigi, R.M. et al. Thin-cap fibroatheroma rupture is associated with a fine interplay of shear and wall stress. Arteriosclerosis, Thrombosis, and Vascular Biology 34, 2224-31 (2014).

43. Gijsen, F., van der Giessen, A., van der Steen, A. \& Wentzel, J. Shear stress and advanced atherosclerosis in human coronary arteries. J Biomech 46, 240-7 (2013). 
44. Davies, P.F., Civelek, M., Fang, Y. \& Fleming, I. The atherosusceptible endothelium: endothelial phenotypes in complex haemodynamic shear stress regions in vivo. Cardiovascular Research 99, 315-327 (2013).

45. Chaudhury, $\mathrm{H}$. et al. c-Jun N-Terminal Kinase Primes Endothelial Cells at Atheroprone Sites for Apoptosis. Arteriosclerosis Thrombosis and Vascular Biology 30, 546-U393 (2010).

46. Heo, K.-S. et al. PKC zeta mediates disturbed flow-induced endothelial apoptosis via p53 SUMOylation. Journal of Cell Biology 193, 867-884 (2011).

47. Magid, R. \& Davies, P.F. Endothelial protein kinase C isoform identity and differential activity of PKC xi in an athero-susceptible region of porcine aorta. Circulation Research 97, 443-449 (2005).

48. Civelek, M., Manduchi, E., Riley, R.J., Stoeckert, C.J., Jr. \& Davies, P.F. Chronic Endoplasmic Reticulum Stress Activates Unfolded Protein Response in Arterial Endothelium in Regions of Susceptibility to Atherosclerosis. Circulation Research 105, 453-U127 (2009).

49. Lee, D.-Y. et al. Role of histone deacetylases in transcription factor regulation and cell cycle modulation in endothelial cells in response to disturbed flow. Proceedings of the National Academy of Sciences of the United States of America 109, 1967-1972 (2012).

50. Schober, A., Nazari-Jahantigh, M. \& Weber, C. MicroRNA-mediated mechanisms of the cellular stress response in atherosclerosis. Nature Reviews Cardiology 12, 361-374 (2015).

51. Warboys, C.M. et al. Disturbed Flow Promotes Endothelial Senescence via a p53Dependent Pathway. Arteriosclerosis Thrombosis and Vascular Biology 34, 985-995 (2014). 
52. Chiu, J.J. et al. Shear stress increases ICAM-1 and decreases VCAM-1 and E-selectin expressions induced by tumor necrosis factor-[alpha] in endothelial cells. Arterioscler Thromb Vasc Biol 24, 73-9 (2004).

53. Nagel, T., Resnick, N., Atkinson, W.J., Dewey, C.F., Jr. \& Gimbrone, M.A., Jr. Shear stress selectively upregulates intercellular adhesion molecule-1 expression in cultured human vascular endothelial cells. J Clin Invest 94, 885-91 (1994).

54. Sheikh, S., Rainger, G.E., Gale, Z., Rahman, M. \& Nash, G.B. Exposure to fluid shear stress modulates the ability of endothelial cells to recruit neutrophils in response to tumor necrosis factor-alpha: a basis for local variations in vascular sensitivity to inflammation. Blood 102, 2828-34 (2003).

55. Matharu, N.M. et al. Inflammatory responses of endothelial cells experiencing reduction in flow after conditioning by shear stress. J Cell Physiol 216, 732-41 (2008).

56. Chappell, D.C., Varner, S.E., Nerem, R.M., Medford, R.M. \& Alexander, R.W. Oscillatory shear stress stimulates adhesion molecule expression in cultured human endothelium. Circ Res 82, 532-9 (1998).

57. Schmitt, M.M.N. et al. Endothelial Junctional Adhesion Molecule-A Guides Monocytes Into Flow-Dependent Predilection Sites of Atherosclerosis. Circulation 129, 66-76 (2014).

58. Hajra, L., Zhu, S.N. \& Cybulsky, M.I. Modulation of NF-kappa B signal transduction in different regions of the arterial tree. Faseb Journal 15, A247-A247 (2001).

59. Cuhlmann, S. et al. Disturbed Blood Flow Induces RelA Expression via c-Jun NTerminal Kinase 1 A Novel Mode of NF-kappa B Regulation That Promotes Arterial Inflammation. Circulation Research 108, 950-959 (2011). 
60. Fang, Y., Shi, C., Manduchi, E., Civelek, M. \& Davies, P.F. MicroRNA-10a regulation of proinflammatory phenotype in athero-susceptible endothelium in vivo and in vitro. Proc Natl Acad Sci U S A 107, 13450-5 (2010).

61. SenBanerjee, S. et al. KLF2 Is a novel transcriptional regulator of endothelial proinflammatory activation. $J$ Exp Med 199, 1305-15 (2004).

62. Yurdagul, A., Jr. et al. Altered nitric oxide production mediates matrix-specific PAK2 and NF-kappaB activation by flow. Mol Biol Cell 24, 398-408 (2013).

63. Partridge, J. et al. Laminar shear stress acts as a switch to regulate divergent functions of NF-kappaB in endothelial cells. FASEB J 21, 3553-61 (2007).

64. Boon, R.A. et al. KLF2 suppresses TGF-beta signaling in endothelium through induction of Smad7 and inhibition of AP-1. Arterioscler Thromb Vasc Biol 27, 532-9 (2007).

65. Fledderus, J.O. et al. Prolonged shear stress and KLF2 suppress constitutive proinflammatory transcription through inhibition of ATF2. Blood 109, 4249-57 (2007).

66. Boon, R.A. et al. KLF2-induced actin shear fibers control both alignment to flow and JNK signaling in vascular endothelium. Blood 115, 2533-42 (2010).

67. Dai, G. et al. Biomechanical forces in atherosclerosis-resistant vascular regions regulate endothelial redox balance via phosphoinositol 3-kinase/Akt-dependent activation of Nrf2. Circ Res 101, 723-33 (2007).

68. Fledderus, J.O. et al. KLF2 primes the antioxidant transcription factor Nrf2 for activation in endothelial cells. Arterioscler Thromb Vasc Biol 28, 1339-46 (2008).

69. Zakkar, M. et al. Activation of Nrf2 in endothelial cells protects arteries from exhibiting a proinflammatory state. Arterioscler Thromb Vasc Biol 29, 1851-7 (2009). 
70. Zakkar, M. et al. Increased endothelial mitogen-activated protein kinase phosphatase1 expression suppresses proinflammatory activation at sites that are resistant to atherosclerosis. Circulation Research 103, 726-732 (2008).

71. Saitoh, M. et al. Mammalian thioredoxin is a direct inhibitor of apoptosis signalregulating kinase (ASK) 1. The EMBO Journal 17, 2596-2606 (1998).

72. Castier, Y., Brandes, R.P., Leseche, G., Tedgui, A. \& Lehoux, S. P47phox-dependent NADPH oxidase regulates flow-induced vascular remodeling. Circulation Research 97, 533-540 (2005).

73. Wang, J.C., Normand, S.-L.T., Mauri, L. \& Kuntz, R.E. Coronary Artery Spatial Distribution of Acute Myocardial Infarction Occlusions. Circulation 110, 278-284 (2004).

74. McDaniel, M.C. et al. Localization of culprit lesions in coronary arteries of patients with ST-segment elevation myocardial infarctions: Relation to bifurcations and curvatures. American heart journal 161, 508-515 (2011).

75. Nam, D. et al. Partial carotid ligation is a model of acutely induced disturbed flow, leading to rapid endothelial dysfunction and atherosclerosis. American Journal of Physiology - Heart and Circulatory Physiology 297, H1535-43 (2009).

76. Phinikaridou, A., Hua, N., Pham, T. \& Hamilton, J.A. Regions of Low Endothelial Shear Stress Colocalize With Positive Vascular Remodeling and Atherosclerotic Plaque Disruption: An In Vivo Magnetic Resonance Imaging Study. Circulation: Cardiovascular Imaging 6, 302-310 (2013).

77. Koskinas, K.C. et al. Natural history of experimental coronary atherosclerosis and vascular remodeling in relation to endothelial shear stress: a serial, in vivo intravascular ultrasound study. Circulation 121, 2092-101 (2010). 
78. Chatzizisis, Y.S. et al. Role of endothelial shear stress in the natural history of coronary atherosclerosis and vascular remodeling: molecular, cellular, and vascular behavior. Journal of the American College of Cardiology 49, 2379-93 (2007).

79. Krams, R. et al. Evaluation of Endothelial Shear Stress and 3D Geometry as Factors Determining the Development of Atherosclerosis and Remodeling in Human Coronary Arteries in Vivo: Combining 3D Reconstruction from Angiography and IVUS (ANGUS) with Computational Fluid Dynamics. Arteriosclerosis, Thrombosis, and Vascular Biology 17, 2061-2065 (1997).

80. Stone, P.H. et al. Effect of Endothelial Shear Stress on the Progression of Coronary Artery Disease, Vascular Remodeling, and In-Stent Restenosis in Humans: In Vivo 6Month Follow-Up Study. Circulation 108, 438-444 (2003).

81. Stone, P.H. et al. Regions of low endothelial shear stress are the sites where coronary plaque progresses and vascular remodelling occurs in humans: an in vivo serial study. European Heart Journal 28, 705-10 (2007).

82. Papafaklis, M.I. et al. Effect of the local hemodynamic environment on the de novo development and progression of eccentric coronary atherosclerosis in humans: Insights from PREDICTION. Atherosclerosis 240, 205-11 (2015).

83. Samady, H. et al. Coronary artery wall shear stress is associated with progression and transformation of atherosclerotic plaque and arterial remodeling in patients with coronary artery disease. Circulation 124, 779-88 (2011).

84. Corban, M.T. et al. Combination of plaque burden, wall shear stress, and plaque phenotype has incremental value for prediction of coronary atherosclerotic plaque progression and vulnerability. Atherosclerosis 232, 271-6 (2014). 
85. Stone, P.H. et al. Prediction of progression of coronary artery disease and clinical outcomes using vascular profiling of endothelial shear stress and arterial plaque characteristics: the PREDICTION Study. Circulation 126, 172-81 (2012).

86. Maurovich-Horvat, P., Ferencik, M., Voros, S., Merkely, B. \& Hoffmann, U. Comprehensive plaque assessment by coronary CT angiography. Nat Rev Cardiol 11, 390-402 (2014).

87. Taylor, C.A., Fonte, T.A. \& Min, J.K. Computational Fluid Dynamics Applied to Cardiac Computed Tomography for Noninvasive Quantification of Fractional Flow ReserveScientific Basis. Journal of the American College of Cardiology 61, 22332241 (2013).

88. Hetterich, H. et al. Coronary Computed Tomography Angiography Based Assessment of Endothelial Shear Stress and Its Association with Atherosclerotic Plaque Distribution In-Vivo. PLoS ONE 10, e0115408 (2015).

89. Burke, A.P. et al. Coronary risk factors and plaque morphology in men with coronary disease who died suddenly. New England Journal of Medicine 336, 1276-82 (1997).

90. Davies, M.J. \& Thomas, A. Thrombosis and acute coronary-artery lesions in sudden cardiac ischemic death. New England Journal of Medicine 310, 1137-40 (1984).

91. Virmani, R., Kolodgie, F.D., Burke, A.P., Farb, A. \& Schwartz, S.M. Lessons from sudden coronary death: a comprehensive morphological classification scheme for atherosclerotic lesions. Arteriosclerosis, Thrombosis and Vascular Biology 20, $1262-$ 75 (2000).

92. Fujii, K. et al. OCT Assessment of Thin-Cap Fibroatheroma Distribution in Native Coronary Arteries. JACC Cardiovascular Imaging 3, 168-175 (2010). 
93. Zhao, Z. et al. Dynamic nature of nonculprit coronary artery lesion morphology in STEMI: a serial IVUS analysis from the HORIZONS-AMI trial. JACC Cardiovascular Imaging 6, 86-95 (2013).

94. Cheng, C. et al. Atherosclerotic lesion size and vulnerability are determined by patterns of fluid shear stress. Circulation 113, 2744-53 (2006).

95. Chatzizisis, Y.S. et al. Prediction of the localization of high-risk coronary atherosclerotic plaques on the basis of low endothelial shear stress: an intravascular ultrasound and histopathology natural history study. Circulation 117, 993-1002 (2008).

96. Traub, O. \& Berk, B.C. Laminar Shear Stress: Mechanisms by Which Endothelial Cells Transduce an Atheroprotective Force. Arteriosclerosis, Thrombosis, and Vascular Biology 18, 677-685 (1998).

97. Tarbell, J.M. Shear stress and the endothelial transport barrier. Cardiovascular research $\mathbf{8 7}, 320-30$ (2010).

98. Mehta, J.L., Chen, J., Hermonat, P.L., Romeo, F. \& Novelli, G. Lectin-like, oxidized low-density lipoprotein receptor-1 (LOX-1): a critical player in the development of atherosclerosis and related disorders. Cardiovascular research 69, 36-45 (2006).

99. Murase, T. et al. Fluid shear stress transcriptionally induces lectin-like oxidized LDL receptor-1 in vascular endothelial cells. Circulation research 83, 328-33 (1998).

100. Koskinas, K.C. et al. Synergistic effect of local endothelial shear stress and systemic hypercholesterolemia on coronary atherosclerotic plaque progression and composition in pigs. International Journal of Cardiology 169, 394-401 (2013).

101. Zhou, J. et al. Regulation of Vascular Smooth Muscle Cell Turnover by Endothelial Cell-Secreted MicroRNA-126: Role of Shear Stress. Circulation research 113, 40-51 (2013). 
102. Qi, Y.X. et al. Rho-GDP dissociation inhibitor alpha downregulated by low shear stress promotes vascular smooth muscle cell migration and apoptosis: a proteomic analysis. Cardiovascular research 80, 114-22 (2008).

103. Koskinas, K.C. et al. Thin-capped atheromata with reduced collagen content in pigs develop in coronary arterial regions exposed to persistently low endothelial shear stress. Arteriosclerosis, Thrombosis and Vascular Biology 33, 1494-504 (2013).

104. Chatzizisis, Y.S. et al. Augmented expression and activity of extracellular matrixdegrading enzymes in regions of low endothelial shear stress colocalize with coronary atheromata with thin fibrous caps in pigs. Circulation 123, 621-30 (2011).

105. Newby, A.C. Metalloproteinase Expression in Monocytes and Macrophages and its Relationship to Atherosclerotic Plaque Instability. Arteriosclerosis, Thrombosis, and Vascular Biology 28, 2108-2114 (2008).

106. Pedrigi, R.M. et al. Inducing Persistent Flow Disturbances Accelerates Atherogenesis and Promotes Thin Cap Fibroatheroma Development in D374Y-PCSK9 Hypercholesterolemic Minipigs. Circulation 132, 1003-1012 (2015).

107. Obaid, D.R. et al. Atherosclerotic plaque composition and classification identified by coronary computed tomography: assessment of computed tomography-generated plaque maps compared with virtual histology intravascular ultrasound and histology. Circulation Cardiovascular Imaging 6, 655-64 (2013).

108. Voros, S. et al. Coronary Atherosclerosis Imaging by Coronary CT Angiography: Current Status, Correlation With Intravascular Interrogation and Meta-Analysis. JACC: Cardiovascular Imaging 4, 537-548 (2011).

109. Pundziute, G. et al. Head-to-Head Comparison of Coronary Plaque Evaluation Between Multislice Computed Tomography and Intravascular Ultrasound 
Radiofrequency Data Analysis. JACC: Cardiovascular Interventions 1, 176-182 (2008).

110. Nair, A., Margolis, M.P., Kuban, B.D. \& Vince, D.G. Automated coronary plaque characterisation with intravascular ultrasound backscatter: ex vivo validation. EuroIntervention 3, 113-20 (2007).

111. Yabushita, H. et al. Characterization of human atherosclerosis by optical coherence tomography. Circulation 106, 1640-5 (2002).

112. Eshtehardi, P. et al. Association of coronary wall shear stress with atherosclerotic plaque burden, composition, and distribution in patients with coronary artery disease. Journal of the American Heart Association 1, e002543 (2012).

113. Wentzel, J.J. et al. In vivo assessment of the relationship between shear stress and necrotic core in early and advanced coronary artery disease. EuroIntervention 9, 98995; discussion 995 (2013).

114. Rodriguez-Granillo, G.A. et al. Plaque Composition and its Relationship With Acknowledged Shear Stress Patterns in Coronary Arteries. Journal of the American College of Cardiology 47, 884-885 (2006).

115. Vergallo, R. et al. Endothelial Shear Stress and Coronary Plaque Characteristics in Humans: A Combined Frequency-Domain Optical Coherence Tomography and Computational Fluid Dynamics Study. Circulation Cardiovascular Imaging (2014).

116. Falk, E. Why do plaques rupture? Circulation 86, III30-42 (1992).

117. Burke, A.P. et al. Healed Plaque Ruptures and Sudden Coronary Death: Evidence That Subclinical Rupture Has a Role in Plaque Progression. Circulation 103, 934-940 (2001). 
118. Loree, H.M., Kamm, R.D., Stringfellow, R.G. \& Lee, R.T. Effects of fibrous cap thickness on peak circumferential stress in model atherosclerotic vessels. Circulation Research 71, 850-8 (1992).

119. Teng, Z. et al. Arterial Luminal Curvature and Fibrous-Cap Thickness Affect Critical Stress Conditions Within Atherosclerotic Plaque: An In Vivo MRI-Based 2D FiniteElement Study. Annals of Biomedical Engineering 38, 3096-3101 (2010).

120. Tang, D., Yang, C., Kobayashi, S. \& Ku, D.N. Effect of a lipid pool on stress/strain distributions in stenotic arteries: 3-D fluid-structure interactions (FSI) models. Journal of Biomechanical Engineering 126, 363-70 (2004).

121. Gao, H. \& Long, Q. Effects of varied lipid core volume and fibrous cap thickness on stress distribution in carotid arterial plaques. Journal of Biomechanics 41, 3053-3059 (2008).

122. Finet, G., Ohayon, J. \& Rioufol, G. Biomechanical interaction between cap thickness, lipid core composition and blood pressure in vulnerable coronary plaque: impact on stability or instability. Coronary Artery Disease 15, 13-20 (2004).

123. Li, Z.Y. et al. Does calcium deposition play a role in the stability of atheroma? Location may be the key. Cerebrovascular Disorders 24, 452-9 (2007).

124. Imoto, K. et al. Longitudinal structural determinants of atherosclerotic plaque vulnerability: a computational analysis of stress distribution using vessel models and three-dimensional intravascular ultrasound imaging. Journal of the American College of Cardiology 46, 1507-15 (2005).

125. Bluestein, D. et al. Influence of microcalcifications on vulnerable plaque mechanics using FSI modeling. Journal of Biomechanics 41, 1111-8 (2008). 
126. Vengrenyuk, Y. et al. A hypothesis for vulnerable plaque rupture due to stressinduced debonding around cellular microcalcifications in thin fibrous caps. Proceedings of the National Academy of Sciences 103, 14678-14683 (2006).

127. Kelly-Arnold, A. et al. Revised microcalcification hypothesis for fibrous cap rupture in human coronary arteries. Proceedings of the National Academy of Sciences 110, 10741-10746 (2013).

128. Irkle, A. et al. Identifying active vascular microcalcification by (18)F-sodium fluoride positron emission tomography. Nature communications 6, 7495 (2015).

129. Tarkin, J.M., Joshi, F.R. \& Rudd, J.H. PET imaging of inflammation in atherosclerosis. Nature Reviews Cardiology 11, 443-57 (2014).

130. Joshi, N.V. et al. F-fluoride positron emission tomography for identification of ruptured and high-risk coronary atherosclerotic plaques: a prospective clinical trial. Lancet 383, 705-703 (2014).

131. Cheng, G.C., Loree, H.M., Kamm, R.D., Fishbein, M.C. \& Lee, R.T. Distribution of circumferential stress in ruptured and stable atherosclerotic lesions. A structural analysis with histopathological correlation. Circulation 87, 1179-87 (1993).

132. Lee, R.T. et al. Computational structural analysis based on intravascular ultrasound imaging before in vitro angioplasty: prediction of plaque fracture locations. Journal of the American College of Cardiology 21, 777-82 (1993).

133. Huang, H. et al. The impact of calcification on the biomechanical stability of atherosclerotic plaques. Circulation 103, 1051-6 (2001).

134. Lee, R.T., Schoen, F.J., Loree, H.M., Lark, M.W. \& Libby, P. Circumferential Stress and Matrix Metalloproteinase 1 in Human Coronary Atherosclerosis: Implications for Plaque Rupture. Arteriosclerosis, Thrombosis and Vascular Biology 16, 1070-1073 (1996). 
135. Hallow, K.M., Taylor, W.R., Rachev, A. \& Vito, R.P. Markers of inflammation collocate with increased wall stress in human coronary arterial plaque. Biomechanics and Modeling in Mechanobiology 8, 473-86 (2009).

136. Qiu, J. et al. Biomechanical regulation of vascular smooth muscle cell functions: from in vitro to in vivo understanding. Journal of the Royal Society Interface 11, 20130852 (2014).

137. Rodriguez, A.I. et al. MEF2B-Nox1 signaling is critical for stretch-induced phenotypic modulation of vascular smooth muscle cells. Arterioscler Thromb Vasc Biol 35, 430-8 (2015).

138. Cattaruzza, M., Dimigen, C., Ehrenreich, H. \& Hecker, M. Stretch-induced endothelin B receptor-mediated apoptosis in vascular smooth muscle cells. FASEB $J \mathbf{1 4}, 991-8$ (2000).

139. Teng, Z. et al. Critical mechanical conditions around neovessels in carotid atherosclerotic plaque may promote intraplaque hemorrhage. Atherosclerosis 223, $321-6(2012)$.

140. Tang, D. et al. 3D MRI-based multicomponent FSI models for atherosclerotic plaques. Annals of Biomedical Engineering 32, 947-60 (2004).

141. Li, Z.Y. et al. Stress analysis of carotid plaque rupture based on in vivo high resolution MRI. Journal of Biomechanics 39, 2611-22 (2006).

142. Li, Z.Y. et al. Structural analysis and magnetic resonance imaging predict plaque vulnerability: a study comparing symptomatic and asymptomatic individuals. Journal of Vascular Surgery 45, 768-75 (2007).

143. Sadat, U. et al. Association between biomechanical structural stresses of atherosclerotic carotid plaques and subsequent ischaemic cerebrovascular events--a 
longitudinal in vivo magnetic resonance imaging-based finite element study. European Journal of Vascular and Endovascular Surgery 40, 485-91 (2010).

144. Ohayon, J., Teppaz, P., Finet, G. \& Rioufol, G. In-vivo prediction of human coronary plaque rupture location using intravascular ultrasound and the finite element method. Coronary Artery Disease 12, 655-63 (2001).

145. Teng, Z. et al. Coronary plaque structural stress is associated with plaque composition and subtype and higher in acute coronary syndrome: the BEACON I (Biomechanical Evaluation of Atheromatous Coronary Arteries) study. Circulation Cardiovascular Imaging 7, 461-70 (2014).

146. Dirksen, M.T., van der Wal, A.C., van den Berg, F.M., van der Loos, C.M. \& Becker, A.E. Distribution of inflammatory cells in atherosclerotic plaques relates to the direction of flow. Circulation 98, 2000-3 (1998).

147. Wentzel, J.J. et al. Endothelial shear stress in the evolution of coronary atherosclerotic plaque and vascular remodelling: current understanding and remaining questions. Cardiovascular research 96, 234-43 (2012).

148. Waxman, S., Ishibashi, F. \& Muller, J.E. Detection and Treatment of Vulnerable Plaques and Vulnerable Patients: Novel Approaches to Prevention of Coronary Events. Circulation 114, 2390-2411 (2006).

149. Sameshima, N. et al. The values of wall shear stress, turbulence kinetic energy and blood pressure gradient are associated with atherosclerotic plaque erosion in rabbits. $J$ Atheroscler Thromb 21, 831-8 (2014).

150. Maalej, N., Holden, J. \& Folts, J. Effect of Shear Stress on Acute Platelet Thrombus Formation in Canine Stenosed Carotid Arteries: An In Vivo Quantitative Study. Journal of Thrombosis and Thrombolysis 5, 231-238 (1998). 
151. Bark, D.L., Jr., Para, A.N. \& Ku, D.N. Correlation of thrombosis growth rate to pathological wall shear rate during platelet accumulation. Biotechnology and bioengineering 109, 2642-50 (2012).

152. Farb, A. et al. Coronary Plaque Erosion Without Rupture Into a Lipid Core: A Frequent Cause of Coronary Thrombosis in Sudden Coronary Death. Circulation 93, 1354-1363 (1996).

153. Arbustini, E. et al. Plaque erosion is a major substrate for coronary thrombosis in acute myocardial infarction. Heart 82, 269-272 (1999).

154. Virmani, R., Burke, A.P. \& Farb, A. Plaque rupture and plaque erosion. Thromb Haemost 1, 1-3 (1999).

155. Fukumoto, Y. et al. Localized Elevation of Shear Stress Is Related to Coronary Plaque Rupture: A 3-Dimensional Intravascular Ultrasound Study With In-Vivo Color Mapping of Shear Stress Distribution. Journal of the American College of Cardiology 51, 645-650 (2008).

156. Gijsen, F.J. et al. Strain distribution over plaques in human coronary arteries relates to shear stress. American Journal of Physiology - Heart and Circulatory Physiology 295, H1608-14 (2008).

157. Bank, A.J., Versluis, A., Dodge, S.M. \& Douglas, W.H. Atherosclerotic plaque rupture: a fatigue process? Medical hypotheses 55, 480-4 (2000).

158. Versluis, A., Bank, A.J. \& Douglas, W.H. Fatigue and plaque rupture in myocardial infarction. Journal of Biomechanics 39, 339-47 (2006).

159. Huang, Y. et al. In vivo MRI-based simulation of fatigue process: a possible trigger for human carotid atherosclerotic plaque rupture. Biomedical Engineering Online 12, $36(2013)$. 
160. Pei, X., Wu, B., Tang, T.Y., Gillard, J.H. \& Li, Z.-Y. Fatigue Crack Growth Under Pulsatile Pressure and Plaque Rupture. JACC: Cardiovascular Imaging 7, 738-740 (2014).

161. Motoyama, S. et al. Multislice Computed Tomographic Characteristics of Coronary Lesions in Acute Coronary Syndromes. Journal of the American College of Cardiology 50, 319-326 (2007).

162. Motoyama, S. et al. Plaque Characterization by Coronary Computed Tomography Angiography and the Likelihood of Acute Coronary Events in Mid-Term Follow-Up. Journal of the American College of Cardiology 66, 337-346 (2015). 